\title{
Influence of climatic factors on fruit morphological traits in Populus euphratica Oliv.
}

\author{
A. Soleimani, V. Etemad, M. Calagari, M. Namiranian, A. Shirvani
}

Soleimani A., Etemad V., Calagari M., Namiranian M., Shirvani A., 2014. Influence of climatic factors on fruit morphological traits in Populus euphratica Oliv.. Ann. For. Res. 57(1): 31-38, 2014.

\begin{abstract}
Populus euphratica Oliv. is a native species in arid and semi- arid zone of Iran distributing naturally in the vast regions. We studied the variation in fruit morphological traits in $P$. euphratica trees originating from seven provenances of Iran. P. euphratica samples were prepared from Karaj Research Station. In this study, catkin length, number of capsules in each catkin, capsule length, capsule width, capsule length to capsule width ratio, number of seeds in each capsule, seed length, seed width, seed length to seed width ratio, the weight of 1000 seeds and germination rate were measured. Analysis of variance on all morphological traits except germination showed significant differences $(P<0.05)$ among provenances. In addition, positive significant correlations were observed in some of the fruit morphological traits. Capsule length, seed length and number of seeds in each capsule showed a significant positive correlation with mean annual precipitation, mean annual temperature and longitude. Seed width showed a significant negative correlation with longitude and latitude. The results show that morphological fruit characteristics in $P$. euphratica are mainly influenced by the mean annual temperature of the origins. Keywords climate, fruit morphological traits, Iran, Populus euphratica.
\end{abstract}

Authors. Azam Soleimani (azamsoleimani.2008@gmail.com) - Department Forestry \& Forest Engineering, College of Natural Resources \& Marine Science, Noor, Mazandaran Province, Iran; Vahid Etemad - Faculty of Natural Resources, University of Tehran, Karaj, Iran; Mohsen Calagari - Research Institute of Forests and Rangelands, Karaj, Iran; Manochehr Namiranian, Anoshirvan Shirvani - Faculty of Natural Resources, University of Tehran, Karaj, Iran.

Manuscript received February 17, 2014; revised June 15, 2014; accepted June 18, 2014; online first June 20, 2014.

\section{Introduction}

Populus euphratica Oliv. has a wide distribution range naturally from west and central Asia to north Africa. The best known feature of this poplar in its areas of occurrence is its strong tolerance to high temperatures and salinity (FAO 1979). This species distributed naturally 
in the vast regions of Iran. It is extended from sub-tropical zone such as, Khuzestan to cold zone such as, Azerbaijan and Zanjan provinces in Iran (Sabeti 1976). The geographical and climatic differences affect the morphological and genetic characteristics of this widely distributed species (Masaka 2003, Ginwal et al. 2005, Tilki \& Alptekin 2005, Alvaninejad et al. 2010). Callaham (1964) studied provenances with the aim to define the genetic and environmental components of phenotypic variability between trees from different geographic origins. Bonilla-Barbosa (1994) studied on seed morphology of 10 species of Nymphaea and showed variation in shape, color, size, ornamentation of sclereids, and trichomes. Donahue (1999) studied geographic variation in leaf, cone and seed morphology of Pinus greggii in native forests that results showed southern populations are morphologically different from the northern populations. Finding out the dissimilarities in different characteristics of $P$. euphratica has therefore been a great interest of many researchers. Study on morphological traits of $P$. euphratica leaves show that there is morphological variations among natural populations (Calagari et al. 2006). Morphological variations in fruit of $P$. euphratica such as, catkin length, capsule length, capsule width, seed length, seed width, number of seed in each capsule, weight of 100 seeds and germination percentage reported in China (Shiji et al. 1996). Saito et al. (2002) studied genetic diversity of Populus euphratica populations in northwestern China by RAPD DNA analysis. Rottenberg et al. (2011) investigated genetic variability in sexually dimorphic and monomorphic populations of Populus euphratica. In addition, the relationship between the variations of the climatic factors of the seed origins and the morphological fruit variations of $P$. euphratica is of great potential in improving growth and promoting seed yield. Several studies have reported the relationships between the variations of the climatic factors and morphological, anatomical as well as physiological characteristics of leaves and seeds (e.g. Hiura 1993, Koike \& Maruyama 1998, Koyama et al. 2002, Calagari et al. 2006, Bayramzadeh et al. 2008, Wen et al. 2011).

The aim of this study was to determine the variation of fruit morphology in the P. euphratica trees with different geographical origins that grown under uniform environmental conditions. In addition, it is supposed that there are relationships between geo-climatic factors of the seed origins and fruit morphological traits in $P$. euphratica. Therefore, this research was based to determine the important geo-climate factors of the original seed sources that related to the fruit morphological traits of $P$. euphratica trees grown under uniform environmental conditions. Also the present study aims at providing basic knowledge regarding the intra-specific variation which may be useful for improvement and regeneration of the species in the nursery.

\section{Materials and methods}

In this study, we used 8 year old $P$. euphratica seedlings grown from the cuts that had been collected from different provenances in Khojir, Lorestan, Golestan, Sarakhs, Dezfol, Gotvand, and Hamidiye and planted in the experimental field of Karaj Research Station ( $35^{\circ} 46^{\prime}$ N, $50^{\circ}$ $56^{\prime}$ E) with mean annual precipitation of 251 $\mathrm{mm}$ and mean annual temperature of $14.1^{\circ} \mathrm{C}$. An eighteen-year meteorological data (19882006) extracted from the records of the nearest meteorological stations for each location were related to the mean annual precipitation and temperature (Table 1). Thirty fully fruits (catkin, capsule and seed, from the middle part of tree crown) were randomly selected from four trees,each of the different provenances and their morphological characteristics were measured. The catkin length (CatL, mm), capsule length (CL, mm) and capsule width (CW, $\mathrm{mm}$ ) were measured with a Vernier with an accuracy of $0.01 \mathrm{~mm}$. Seed length (SL, mm), and 
seed width (SW, mm) were measured with a binocular $(10 \mathrm{X})$ (Figure 1). Number of capsules in each catkin (NC) and number of seeds in each capsule (NS) were counted. Weight of 1000 seeds (WS, gr) was measured with a Sartorius balance $( \pm$.00001). Germination rate were determined using ISTA method (ISTA 1993). Data were analyzed using one-way ANOVA to determine differences of fruit ori- gins. Mean separations were performed using Duncan's multiple range test $(P<0.05)$. Correlation analysis was used to determine the linear relationship between the geographical factors and fruit morphological traits. Morphological variations among origin trees were obtained by using principal components analysis (PCA). All data analysis was performed with the softwares SPSS version 18.0 and SAS version 6.03.

Table 1 Climatic factors of $P$. euphratica provenances

\begin{tabular}{|c|c|c|c|c|c|}
\hline Locality & $\begin{array}{l}\text { Mean annual precipitation } \\
(\mathrm{mm})\end{array}$ & $\begin{array}{l}\text { Mean annual temperature } \\
\left({ }^{\circ} \mathrm{C}\right)\end{array}$ & $\begin{array}{l}\text { Altitude } \\
\text { (m) }\end{array}$ & Longitude & Latitude \\
\hline Hamidiyeh & 194.5 & 24.2 & 55 & $48^{\circ} 25^{\prime} \mathrm{E}$ & $31^{\circ} 30^{\prime} \mathrm{N}$ \\
\hline Golestan & 201.9 & 17.1 & 50 & $54^{\circ} 56^{\prime} \mathrm{E}$ & $37^{\circ} 16^{\prime} \mathrm{N}$ \\
\hline Gotvand & 295.9 & 24.8 & 80 & $48^{\circ} 52^{\prime} \mathrm{E}$ & $32^{\circ} 08^{\prime} \mathrm{N}$ \\
\hline Dezfol & 444.3 & 24.0 & 140 & $48^{\circ} 20^{\prime} \mathrm{E}$ & $32^{\circ} 15^{\prime} \mathrm{N}$ \\
\hline Sarakhs & 203.3 & 17.6 & 260 & $61^{\circ} 10^{\prime} \mathrm{E}$ & $36^{\circ} 15^{\prime} \mathrm{N}$ \\
\hline Lorestan & 523.1 & 22.9 & 850 & $47^{\circ} 55^{\prime} \mathrm{E}$ & $32^{\circ} 15^{\prime} \mathrm{N}$ \\
\hline Khojir & 231.9 & 17.6 & 1320 & $51^{\circ} 45^{\prime} \mathrm{E}$ & $35^{\circ} 39^{\prime} \mathrm{N}$ \\
\hline
\end{tabular}

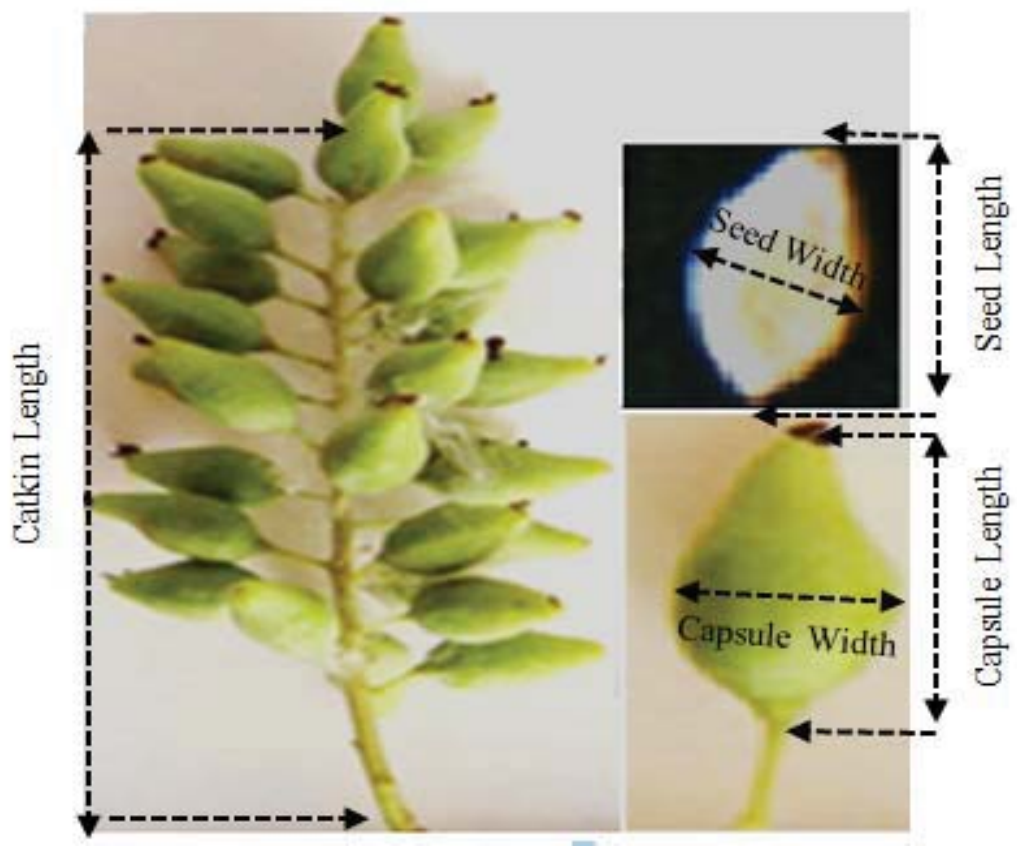

Figure 1 Diagram of fruit morphological trait of $P$. euphratica: Catkin length (CatL), Capsul length (CL), Capsul width (CW), Seed length (SL) and Seed width (SW) 


\section{Results}

\section{Morphological traits}

Analysis of variance on fruit morphological traits showed significant differences $(P<0.01)$ among provenances while germination percentage data showed non-significant difference $(P>0.05)$ among provenances. Mean separations showed $P$. euphratica different ranking traits (Figure 2). Gotvand had the largest CatL (62.48 mm), WS (0.091 gr) and SL (1.09 mm), while Sarakhs showed the smallest CatL, WS and SL (38.01 mm, 0.03gr, and $0.8 \mathrm{~mm}$ respectively). Dezfol showed the highest values for CL, CW and CL/CW, which were $13.68 \mathrm{~mm}$, $5.66 \mathrm{~mm}$ and 2.44, respectively. Minimum and maximum values for SW were belong to Sarakhs $(0.4 \mathrm{~mm})$ and Hamidiyeh $(0.64 \mathrm{~mm})$. Sarakhs had the largest NS and SL/SW (117.62 and 2.0, respectively) while Lorestan indicated the smallest NS (49.07), but the smallest SL/SW (1.50) belonged to Khojir. Lorestan and Hamidiyeh had the maximum and minimum values for $N C$ (29.8 and 19.57, respectively). The high germination percentages are observed in Gotvand, Hamidiye and Lorestan (99\%), while the low germination percentage is seen in Golestan (95.67\%).

\section{Correlations between morphological traits}

There is a good linear relationship among some of the fruit morphological traits and between fruit morphological traits and climatic factors (Table 2). Capsule width positively correlates $(P<0.01)$ with capsules number of catkin, and negatively with capsule length. However the correlation of capsule length with capsule width ( $r=0.53)$ is stronger than with capsules number of catkin $(r=-0.24)$. The parameters catkin length, capsules number of catkin and capsule length correlate with seed length ( $p$ $<0.05)$. There is a strong linear relationship between 1000 seed weight and seed length ( $r$ $=0.66$ ). But the correlation between WS and
NC is week ( $r=-0.15)$. According to Table 2, almost all the parameters of the morphological traits show no significant correlation with altitude. There is a high negative correlation between SW and latitude as well as between SW and longitude ( $r=-0.913$ and -0.796 , respectively). SL show positive trends against mean annual temperature and mean annual precipitations. There is a strong correlation between NS and longitude ( $r=0.819$ ) as well as the correlation between CL and mean annual temperature ( $r=0.757)$. However, the linear relationships between Ns, CL and others fruit morphological traits are fairly weak.

\section{Multivariate analysis}

Principal component analysis reduced 11 variables to two principal components (PC). The result of the PCA indicated four groups (Figure 3). The first two principal components explained $72 \%$ of the total variation. The first and second principal components explained $48 \%$ and $24 \%$ of the total variation, respectively (Table 3). Scores in PC1 were positively related to CatL, SL, SW, CL/CW, WS and GR as well as negatively related to NS. Scores in PC2 were positively related to CL and WC as well as negatively related to NC and SL/SW. Provenances of Dezfoul, Hamidiye were as the first group, Khojir as the second, Gotvand and Lorestan as the third and other provenances such as Golestan and Sarakhs as fourth group.

\section{Discussions}

In this study, the result showed a high level of morphological variability among populations of $P$. euphratica with different provenances. The morphological traits of fruits are one of the most important characteristics for inter and intra-specific diversity studies. As mentioned earlier, fruit morphological traits of the samples which were obtained from different provenances and grown under same environmen- 

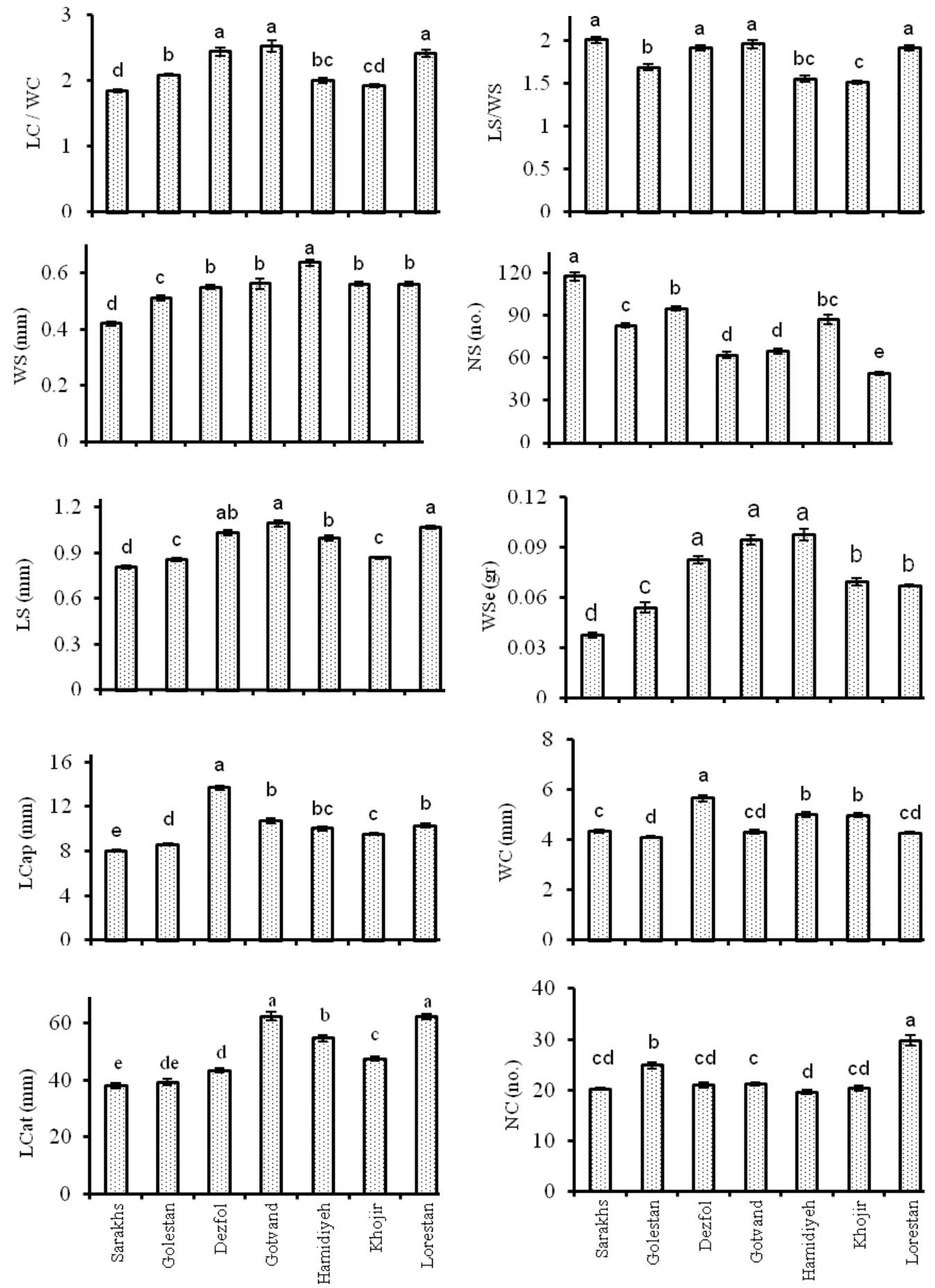

Figure 2 Different fruit traits in the seven provenances of $p$. euphratica. Means with the same letters are not significantly different (ANOVA, $P<0.05)$

tal conditions showed significant differences, with the exception of germination rate (Figure 1). Therefore, it can be suggested that, catkin length, number of capsules in each catkin, capsule length, capsule width, capsule length to capsule width ratio, number of seeds in each capsule, seed length, seed width, seed length to seed width ratio, weight of 1000 seeds in $p$. euphratica are genetically variable.

Shiji et al. (1996) reported catkin length $P$. 
Table 2 Correlation coefficients between fruit morphological traits and the environmental factors (altitude (Alt), longitude (Long), latitude (Lat), mean annual temperature (Mat) and mean annual precipitation (Map)) in studied provenances of $P$. euphratica

\begin{tabular}{lllllllllll}
\hline Traits & CatL & NC & CL & CW & SL & SW & WS & NS & CL/CW & SL/SW \\
\hline NC & $0.200^{* *}$ & \multicolumn{1}{l}{ C } & & & & & & & & \\
CL & $0.210^{* *}$ & -0.080 & & & & & & & & \\
CW & -0.030 & $-0.240^{* *}$ & $0.530^{* *}$ & & & & & & & \\
SL & $0.560^{* *}$ & $0.530^{* *}$ & $0.530^{* *}$ & 0.080 & & & & & & \\
SW & $0.430^{* *}$ & -0.060 & $0.310^{* *}$ & $0.270^{* *}$ & $0.500^{* *}$ & & & & & \\
WS & $0.430^{* *}$ & -0.150 & $0.490^{* *}$ & $0.370^{* *}$ & $0.660^{* *}$ & $0.630^{* *}$ & & & & \\
NS & $-0.570^{* *}$ & -0.140 & $-0.140^{*}$ & $0.150^{*}$ & $-0.530^{* *}$ & $-0.490^{* *}$ & $-0.480^{* *}$ & & & \\
CL/CW & $0.340^{* *}$ & 0.100 & $0.66^{* *}$ & $-0.260^{* *}$ & $0.550^{* *}$ & $0.150^{*}$ & $0.300^{*}$ & $-0.360^{* *}$ & & \\
SL/SW & -0.003 & 0.080 & 0.097 & $-0.210^{* *}$ & $0.250^{* *}$ & $-0.66^{* *}$ & -0.070 & 0.089 & $0.280^{* *}$ & \\
Alt & -0.245 & -0.581 & -0.501 & -0.278 & 0.468 & -0.169 & 0.262 & -0.364 & -0.277 & -0.373 \\
Long & -0.685 & 0.152 & -0.642 & -0.274 & -0.760 & $-0.796^{*}$ & -0.413 & $0.819 *$ & 0.454 & -0.6 \\
Lat & -0.57 & 0.149 & 0.652 & -0.28 & -0.59 & $-0.913^{* *}$ & -0.309 & 0.603 & -0.462 & -0.236 \\
Mat & 0.621 & -0.035 & $0.757^{*}$ & 0.311 & $0.90^{* *}$ & 0.154 & 0.486 & -0.487 & 0.564 & 0.385 \\
Map & 0.511 & -0.273 & 0.410 & 0.404 & $0.763^{*}$ & -0.002 & 0.119 & -0.425 & 0.003 & 0.263 \\
\hline
\end{tabular}

Note. ** , * Significant at $(P<0.01)$ and $(P<0.05)$ levels of probability, respectively

Table 3 Principal components (PC) of fruit morphological traits from different provenances of P. euphratica

\begin{tabular}{lcl}
\hline Trait & PC1 & PC2 \\
\hline CatL & 0.3855 & - \\
CL & - & 0.3441 \\
CW & - & 0.5142 \\
CL/CW & 0.3070 & - \\
NC & -0.4673 \\
SL & 0.4064 & - \\
SW & 0.3534 & - \\
SL/SW & - & -0.3611 \\
NS & -0.3679 & - \\
WS & 0.3843 & - \\
GR & 0.2678 & - \\
Explained variance (\%) & 48.2400 & 24.0500 \\
\hline
\end{tabular}

euphratica in China is about $3-6 \mathrm{~cm}$ that is nearly similar to this study $(3.8-6.2 \mathrm{~cm})$. Capsule length $(8-13.6 \mathrm{~mm})$ in this study is more than reported by Shiji et al. (1996). Capsule width (4.1-5.6 mm) is similar to results of Shiji et al. (1996). Shiji et al. (1996) reported number of seeds in capsules varies from 20 to 25, but in this study number of seeds per capsules varies from 49 to 117. Seeds of $P$. euphratica in Iran are similar to seeds of $P$. euphratica in China (Shiji et al. 1996) morphologically but 1000 seeds weight of Iran is significantly lower. Results of germination percentage in this study are similar to reported results by Shiji et al. (1996).

Variations in fruit morphological traits be- tween different provenances of P. euphratica may be caused by geo-climatic factors. The result shows the relationships between climatic factors and SW, NS, CL and SL. Studies showed that longitude and latitude were different among the provenances. Our sampling covered a broad latitudinal ( $31^{\circ} 30^{\prime}-37^{\circ} 16^{\prime}$ $\mathrm{N})$ and longitudinal $\left(47^{\circ} 55^{\prime} \mathrm{E}-61^{\circ} 10^{\prime} \mathrm{E}\right)$ ranges. SW and NS of the samples showed a significant correlation with the latitude and longitude of the original provenances (Table 2). Therefore, it can be noted that SW and NS in $P$. euphratica are genetically variable against longitude and latitude of the origins. Therefore SW and NS were influenced by latitude and longitude and these traits persisted, 


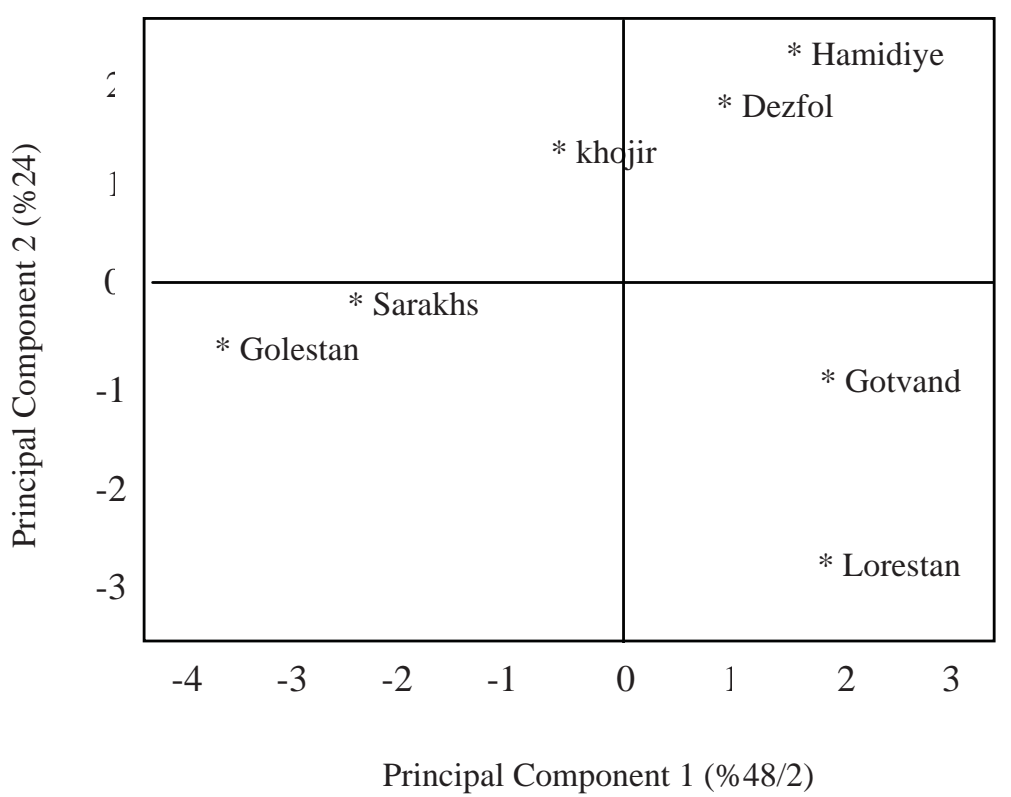

Figure 2 Different fruit traits in the seven provenances of $p$. euphratica. Means with the same letters are not significantly different (ANOVA, $P<0.05$ )

as they could be genetically fixed. The fruit morphological traits showed non-significant relationship with altitude of the original provenances. Thus, the fruit morphological traits in $P$. euphratica are not genetically variable against the altitude of the origins. The highly correlated relationships between mean annual temperature with CL and LS revealed that CL and SL were influenced by the temperatures in $P$. euphratica and this correlations may indicate the adapting capability of $P$. euphratica to a wide range of temperature. SL showed positive trends against mean annual precipitation. Detailed studies on the mean annual precipitation of the provenances showed that the amount of mean annual precipitation differed among the provenances (Table 1 ). The range of mean annual precipitation was between 194.5 mm (Hamidiye) and $523.1 \mathrm{~mm}$ (Lorestan). Correlation among some of leaf morphological traits such leaf thickness with mean annual temperature or petiole length with longitude reported on $P$. eupratica (Calagari et al. 2006). According to the results, fruit morphological traits in P. euphratica are influenced by climatic and geographic factors of the original provenances and the mean annual temperature is the most important factor. Therefore it can be said that the provenances of $P$. euphratica are adapted to the environmental conditions of Iran. But, multisite common field experiments would be needed for generalize the findings for $P$. euphratica in Iran.

\section{Conclusions}

Fruit morphological traits of the samples which were prepared from different provenances and grown under uniform environmental conditions showed significant differences. Therefore, it can be suggested that, mentioned factors in $P$. euphratica are genetically variable. According to the results, fruit morphological traits in $P$. euphratica are influenced by climatic and geographic factors of the original provenances and the mean annual temperature is the most important factor in this regard. Multivariate analysis results revealed four distinct groups of provenances. 


\section{References}

Alvaninejad S., Tabari M., Espahbodi K., Taghvaei M., Hamzepour M., 2010. Morphology and germination characteristics of Quercus brantii Lindl. Acorns in nursery. Iranian Journal of Forest and Poplar Research 17 (4): 523-533.

Bayramzadeh V., Funada R., Kubo T., 2008. Relationships between vessel element anatomy and physiological as well as morphological traits of leaves in Fagus crenata seedlings originating from different provenances. Trees - Structure and Function 22: 217-224.

Bonilla-Barbosa J., Novelo A., Hornelas Orozco Y., Marquez-Guzman J., 2000. Comparative seed morphology of Mexican Nymphaea species. Aquatic Botany 68: 189-204. DOI: 10.1016/S0304-3770(00)00125-X.

Calagari M., Modir-Rahmati A., Asadi F., 2006. Morphological variation in leaf traits of Populus euphratica Oliv. Natural population. International Journal of Agriculture and Biology 8(6): 754-758.

Callaham R.Z., 1964. Provenance research: Investigation of genetic diversity associated with geography. Unasylva 18: 40-50.

Donahue J.K., Lopez Upton J., 1996. Geographic variation in leaf, con and seed morphology of pinus greggii in navite forests. Forest Ecology and Management. 82: 145-157. DOI: 10.1016/0378-1127(95)03677-6.

FAO, 1979. Poplar and willow in wood production and land use. FAO Forest Service 10: 328-329.

Ginwal H.S., Phartyal S.S., Rawat P.S., Srivastava R.L., 2005. Seed source variation in morphology, germination and seedling growth of Jatropha curcas Linn. in Central India. Silvae Genetica 54(2): 76-80.

Hiura T., 1993. Geographical variation and maintainance of species diversity of beech forest in Japan. Japanese Journal of Ecology. 46: 175-178.

ISTA, 1993. International rules for seed testing. Seed Science Technology 21: 1-288.

KoikeT., MaruyamaY., 1998. Comparative ecophysiolᄀogy of the leaf photosynthetic traits in Japanese beech grown in provenances facing the Pacific Ocean side and the sea side of Japan. Journal of Phytogeogrography and Taxonomy. 46: 23-28.

Koyama Y., Yagihashi T., Migita C., Tanaka N., 2002. Geographical gradient of leaf area of Fagus crenata in the Nagano region, Central Japan. Japanese Journal of Forest Environment. 44: 31-33.

Masaka K., 2003. Preliminary Study of Geographic Trends in Acorn Mass and Seedling Emergence Behavior of Quercus dentate in Hokkaido, Northern, Japan. Hokkaido Forestry Research Institute, Bibai, Hokkaido, Japan. 30 p.

Rottenberg A., Nevo E., Zohary D., 2011. Genetic variability in sexually dimorphic and monomorphic populations of Populus euphratica (Salicaceae). Canadian Journal of Forest Research 30(3): 482-486. DOI: 10.1139/cjfr-30-3-482.

Sabeti H., 1976. Forests, trees and shrubs of Iran. Research Institute of Forests and Rangelands Publishers, Tehran, Iran, $806 \mathrm{p}$.

Saito Y., Shiraishi S., Tanimoto T., Yin L., Watanabe S., Ide Y., 2002. Genetic diversity of Populus euphratica populations in northwestern China determined by RAPD DNA analysis. New Forests 23: 97-103. DOI: 10.1023/A:1015605928414.

Shiji W., Binghao C., Hugun L., 1996. Euphrates poplar forest. China Environmental Science Press, Beijing. $117 \mathrm{p}$.

Tilki F., Alptekin C.U., 2005. Variation in acorn characteristics in provenances of Quercus aucheri Jaub. et Spach and provenance, temperature and storage effects on acorn germination. Seed Science Technology 33: 441-447.

Wen Y., Tanga M., Sun D., Zhu H., Wei J., Chen F., Tang L., 2012. Influence of Climatic Factors and Soil Types on Seed Weight and Oil Content of Jatropha curcas in Guangxi, China. Procedia Environmental Sciences. 12: 439-444. DOI: 10.1016/j.proenv.2012.01.301. 